\title{
Effects of the alternating backbone configuration on the secondary structure and self-assembly of $\beta$-peptides
}

\author{
Tamás A. Martinek, ${ }^{\dagger}$ István M. Mándity, ${ }^{\dagger}$ Lívia Fülöp, ${ }^{\ddagger}$ Gábor K. Tóth, ${ }^{\ddagger}$ Elemér Vass, ${ }^{\S}$ \\ Miklós Hollósi, ${ }^{\S}$ Enikö Forró, ${ }^{\dagger}$ Ferenc Fülöp ${ }^{\dagger, *}$
}

Contribution from the Institute of Pharmaceutical Chemistry, Department of Medical Chemistry, University of Szeged, H-6701 Szeged, POB 427, Hungary, and Institute of Chemistry, Department of Organic Chemistry, Eötvös Loránd University, Pázmány P. s. 1/A, 1117 Budapest, Hungary AUTHOR EMAIL ADDRESS: fulop@ pharm.u-szeged.hu

\author{
SUPPORTING INFORMATION \\ TABLE OF CONTENTS
}

Title

Page

Table S1: NMR signal assignment of the backbone for 2 in DMSO and $\mathrm{CD}_{3} \mathrm{OH}(\mathrm{ppm})$

Figures S1-8: ROESY and TOCSY spectra of 1-4 recorded in DMSO

$\mathrm{Ab}$ initio computational data

Complete ref 16 
Table S1. NMR signal assignment of the backbone for 2 in DMSO and $\mathrm{CD}_{3} \mathrm{OH}(\mathrm{ppm})$

\begin{tabular}{c|ccc|ccc|} 
& \multicolumn{3}{|c|}{$\mathbf{D M S O}^{\mathbf{2}}$} & \multicolumn{3}{|c}{$\mathbf{C D}_{\mathbf{3}} \mathbf{O H}$} \\
$\boldsymbol{i}$ & $\mathbf{N H}_{\boldsymbol{i}}$ & $\mathbf{C}^{\boldsymbol{\alpha}} \mathbf{H}_{\boldsymbol{i}}$ & $\mathbf{C}^{\boldsymbol{\beta}} \mathbf{H}_{\boldsymbol{i}}$ & $\mathbf{N H}_{\boldsymbol{i}}$ & $\mathbf{C}^{\boldsymbol{a}} \mathbf{H}_{\boldsymbol{i}}$ & $\mathbf{C}^{\boldsymbol{\beta}} \mathbf{H}_{\boldsymbol{i}}$ \\
\hline 1 & 7.78 & 2.92 & 3.74 & 7.89 & 2.99 & 3.87 \\
2 & 8.31 & 2.74 & 4.39 & 8.26 & 2.90 & 4.53 \\
3 & 8.70 & 2.69 & 4.24 & 8.76 & 2.81 & 4.34 \\
4 & 8.15 & 2.85 & 4.52 & 8.07 & 2.95 & 4.62 \\
5 & 8.38 & 2.54 & 4.32 & 8.21 & 2.70 & 4.42 \\
6 & 8.05 & 2.72 & 4.44 & 8.42 & 2.91 & 4.54 \\
\hline
\end{tabular}




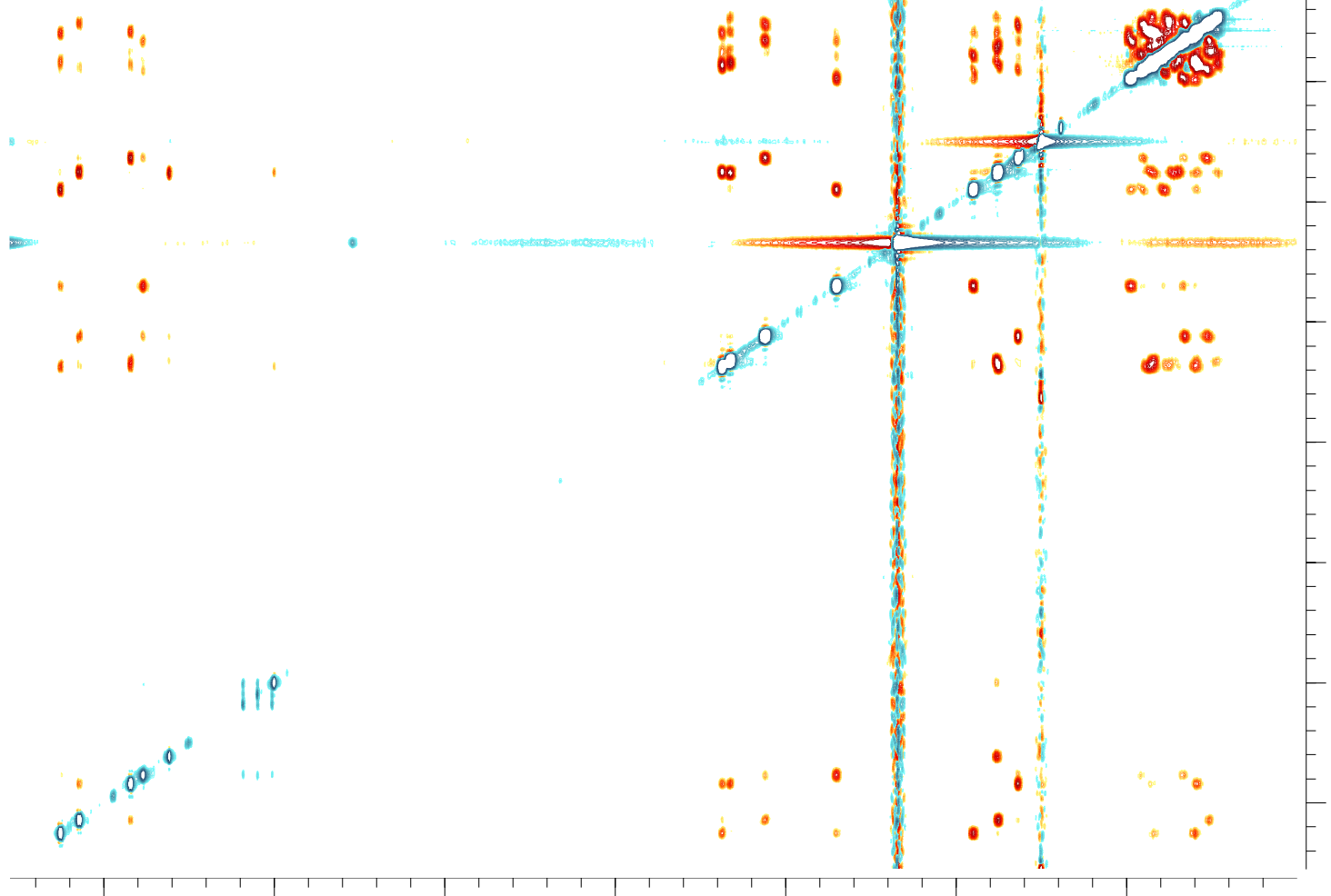

Figure S1. ROESY spectrum of 1 recorded in DMSO

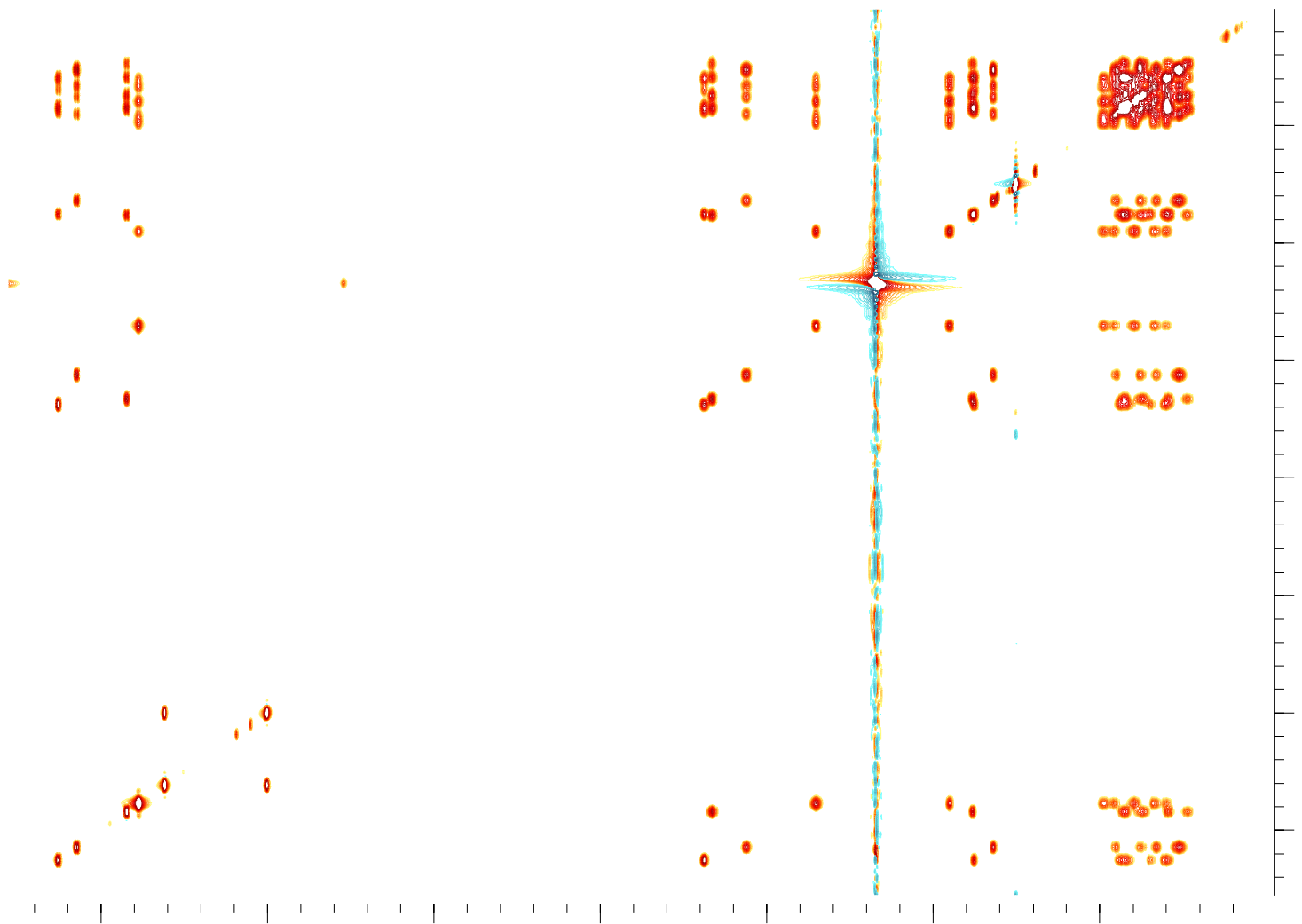

Figure S2. TOCSY spectrum of 1 recorded in DMSO 


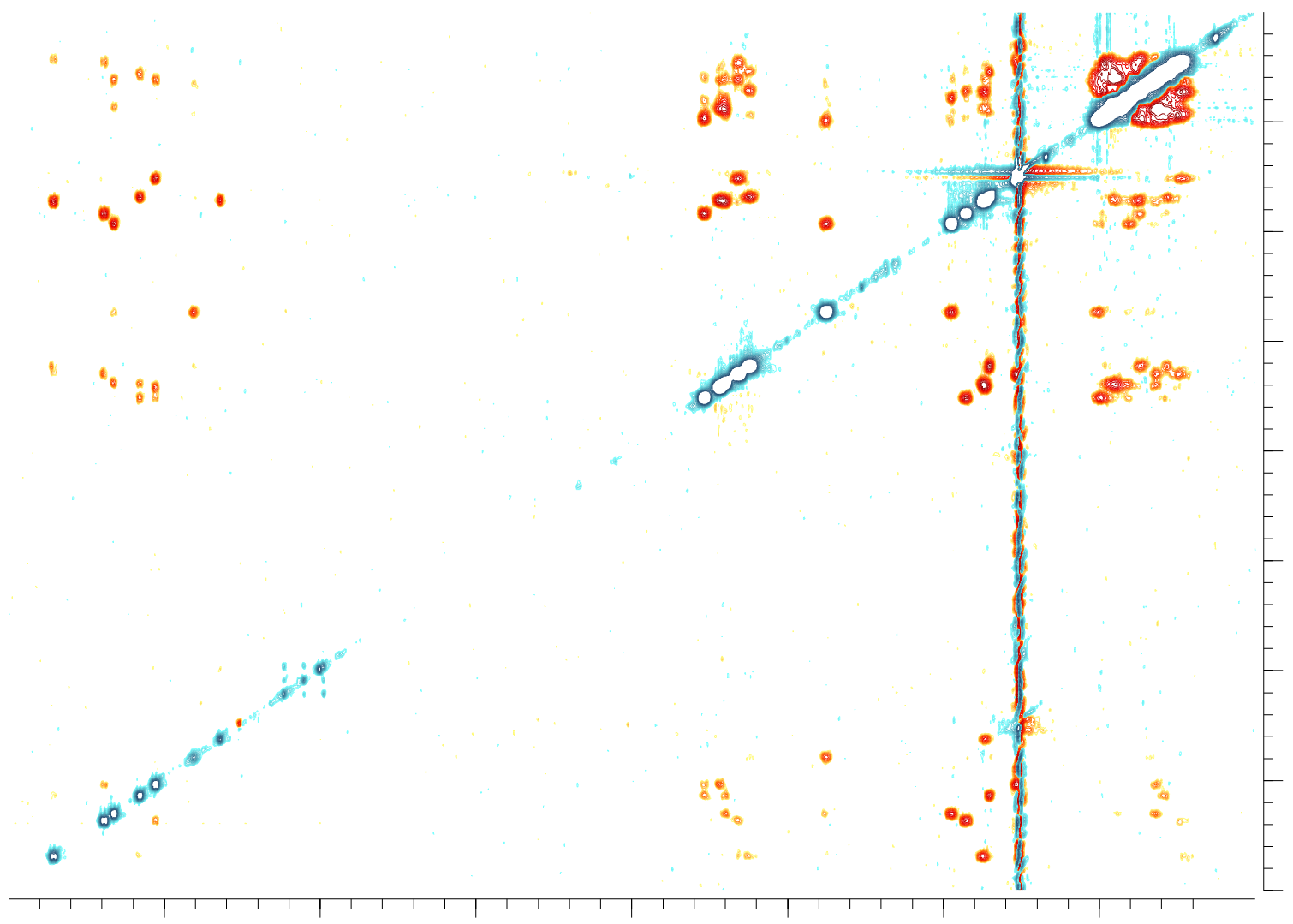

Figure S3. ROESY spectrum of 2 recorded in DMSO

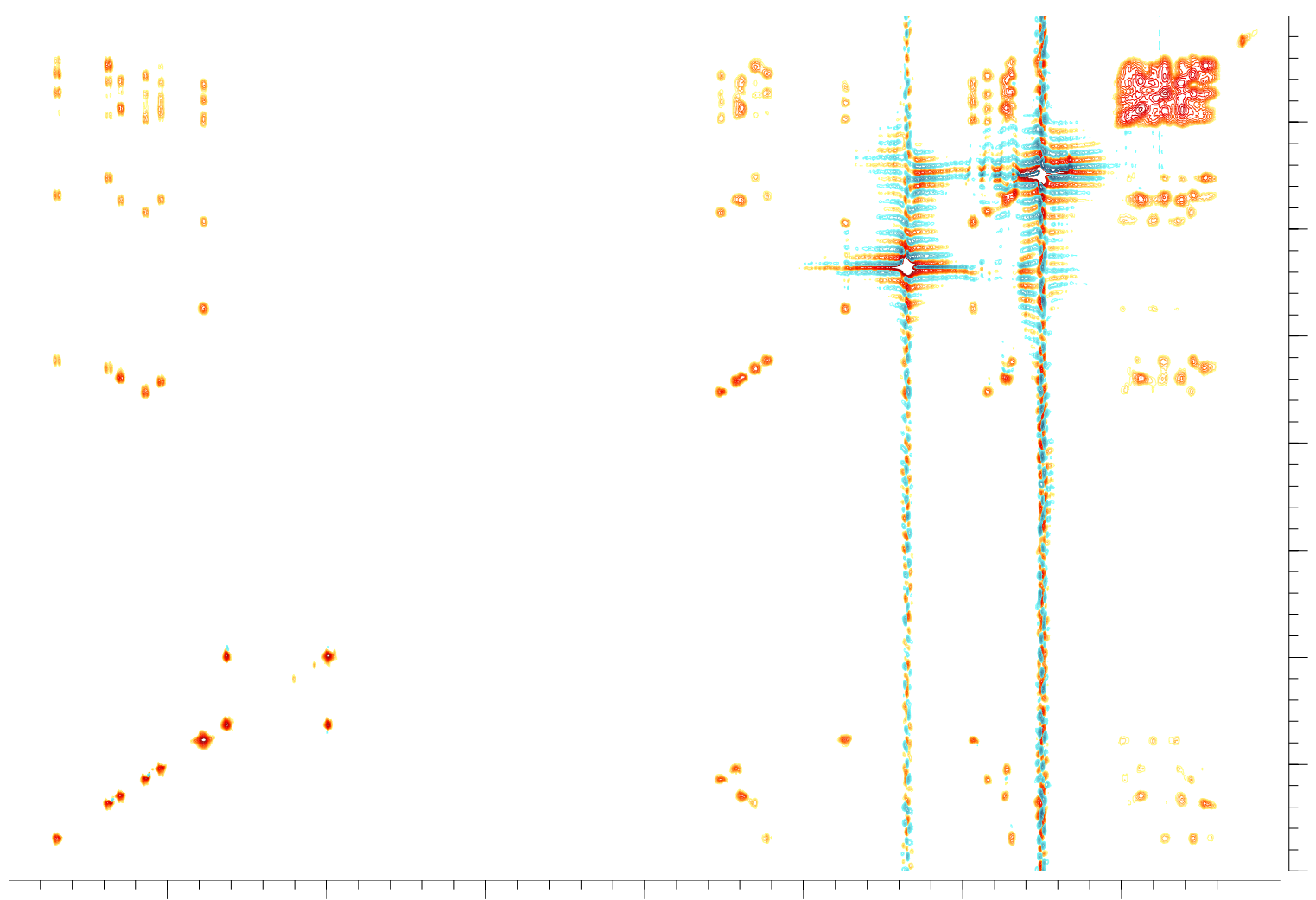

Figure S4. TOCSY spectrum of 2 recorded in DMSO 


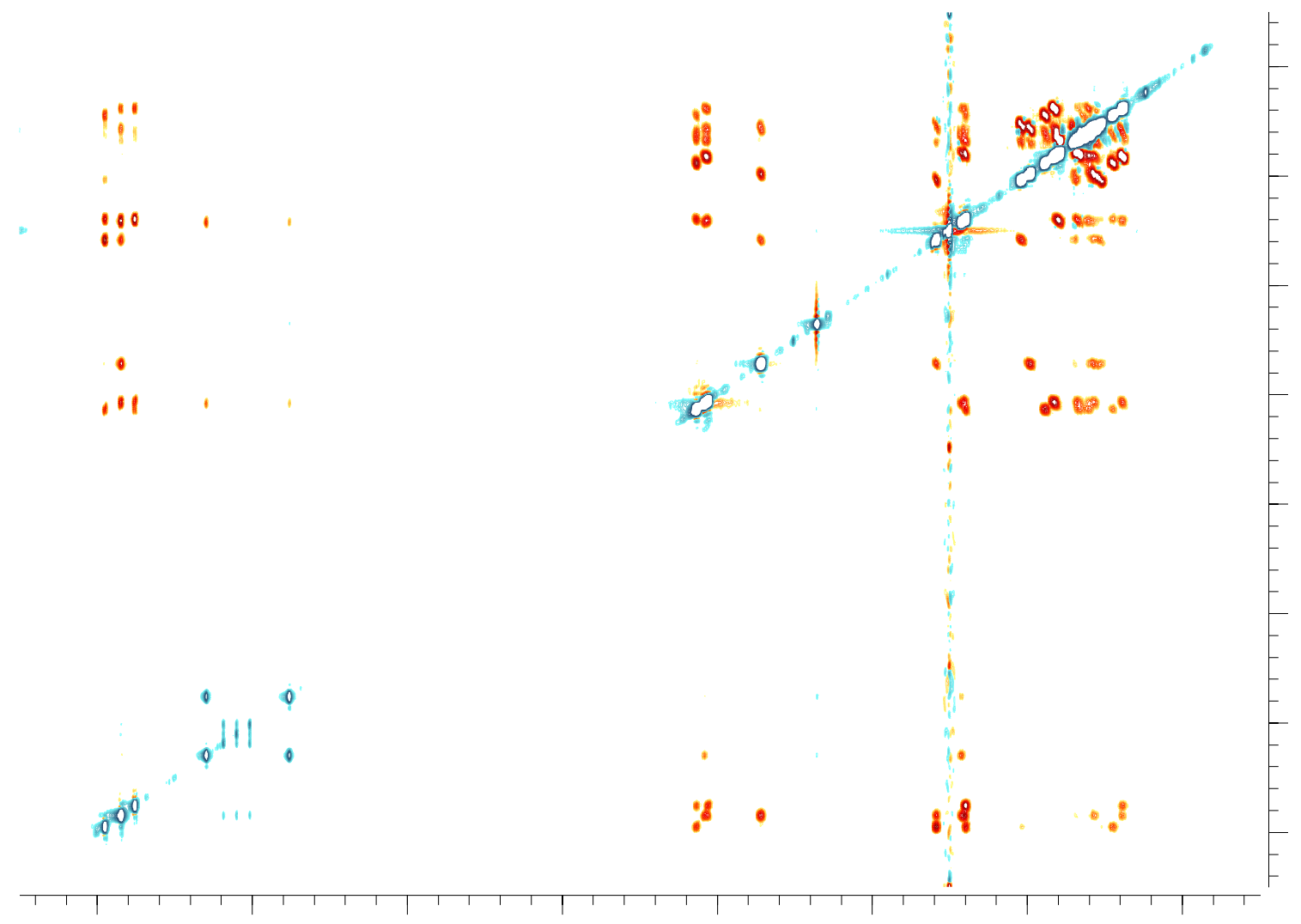

Figure S5. ROESY spectrum of 3 recorded in DMSO

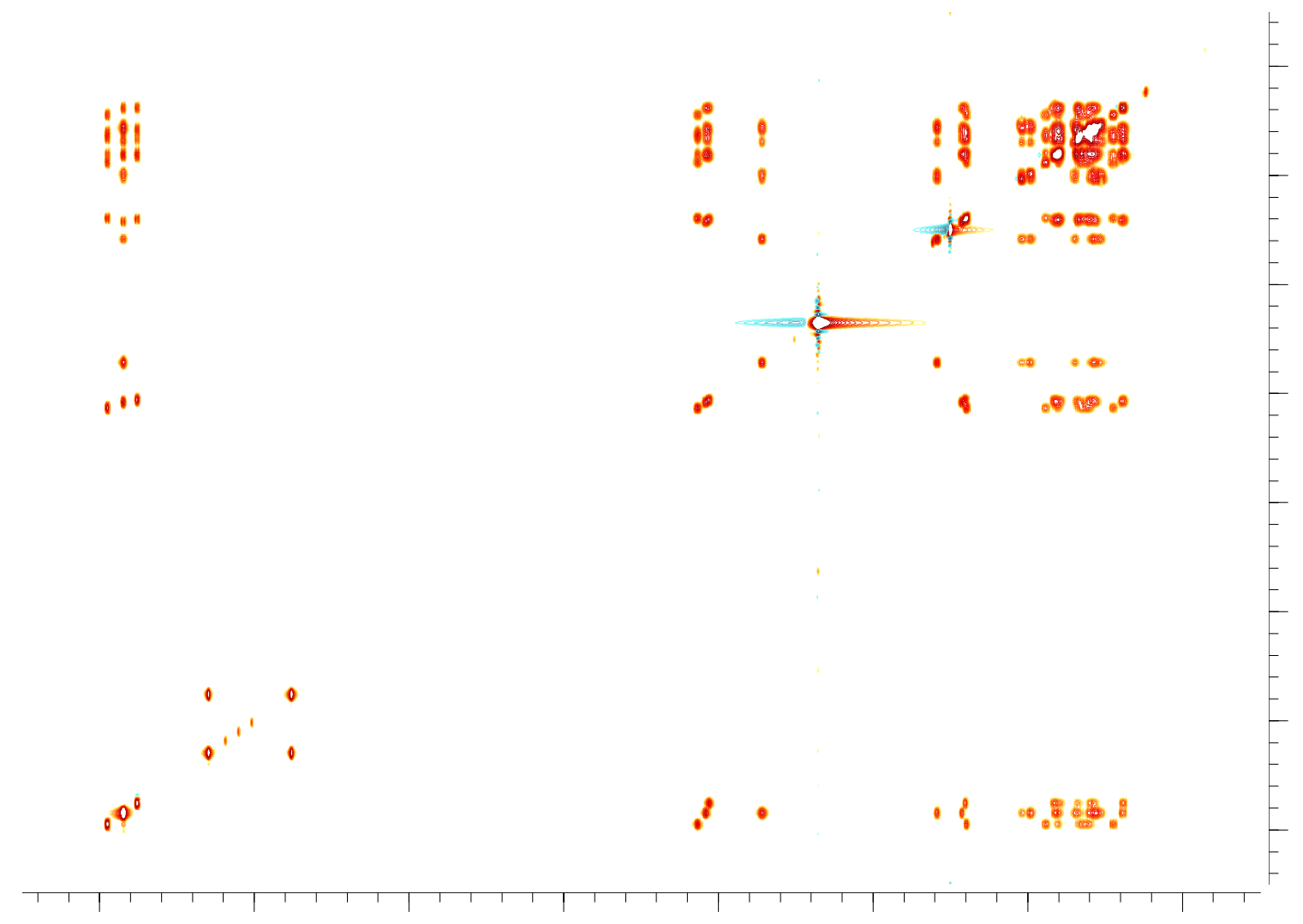

Figure S6. TOCSY spectrum of 3 recorded in DMSO 


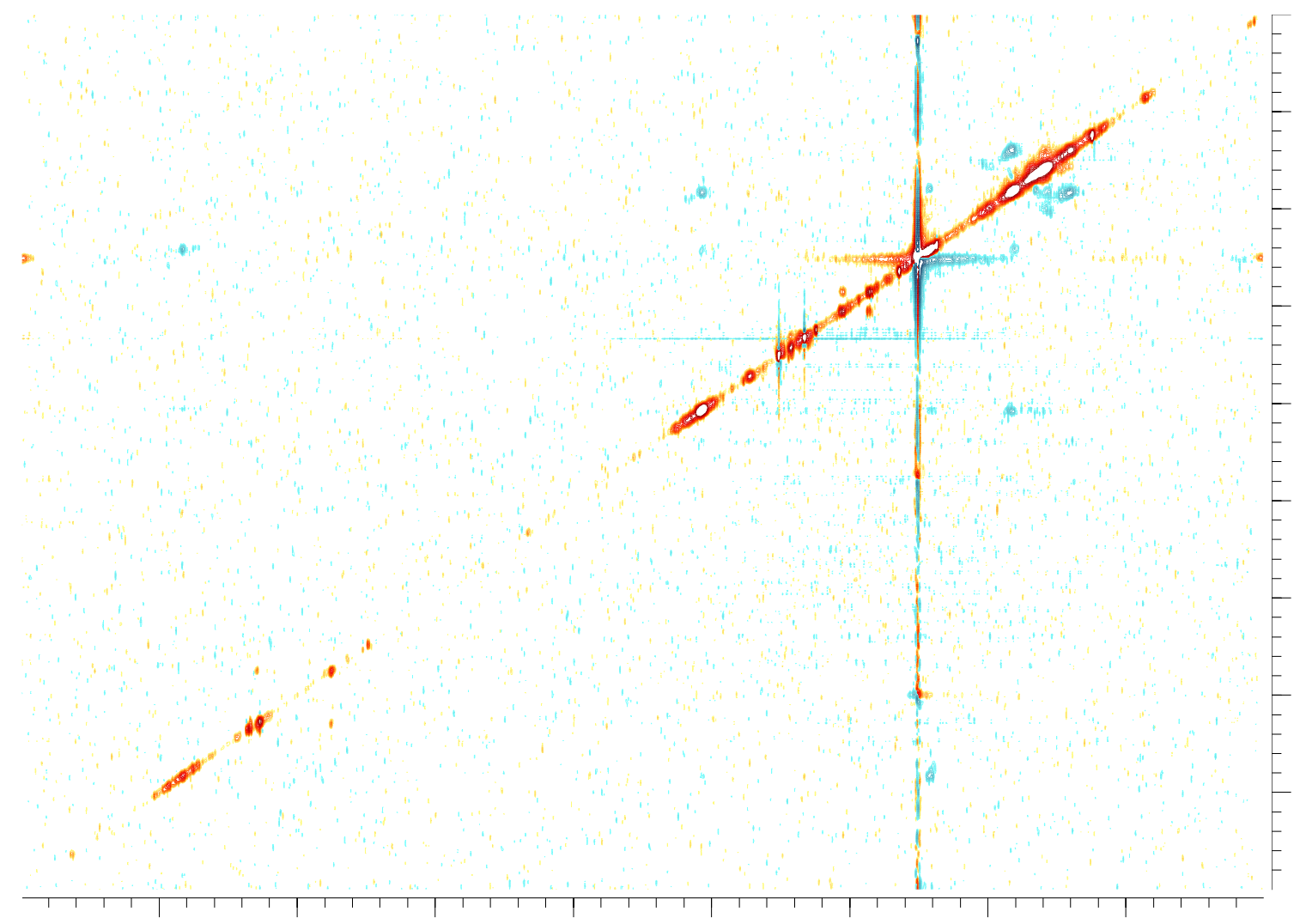

Figure S7. ROESY spectrum of $\mathbf{4}$ recorded in DMSO

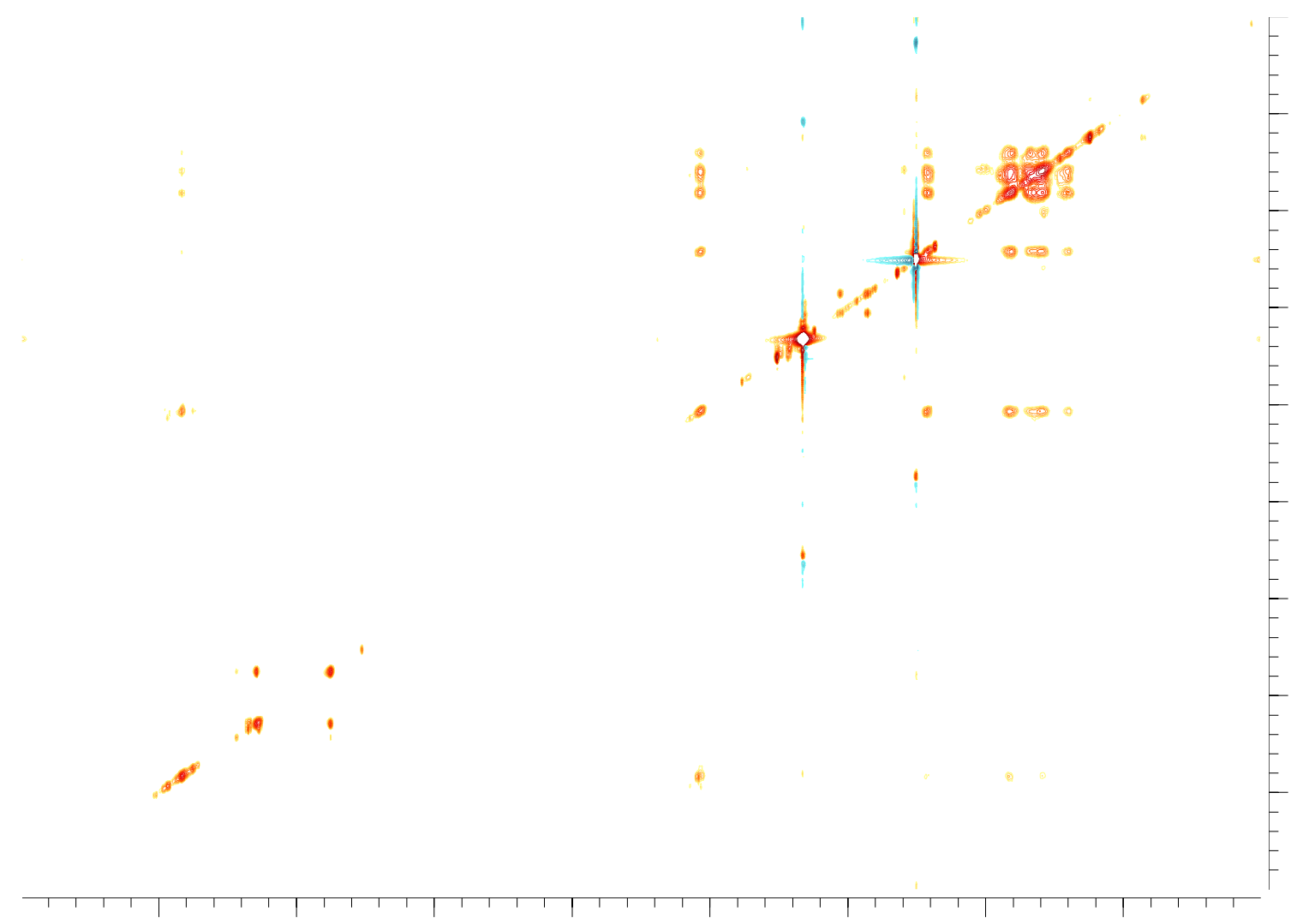

Figure S8. TOCS spectrum of $\mathbf{4}$ recorded in DMSO 
Computational Data

(at the B3LYP/6-311G** level):

2: $\mathrm{H} 10 / 12$

B3LYP/6-311G**: -2241.54603035 a. u.

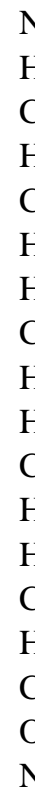

$\mathrm{H}$

$\mathrm{C}$

$\mathrm{H}$

C

$\mathrm{H}$

$\mathrm{H}$

C

$\mathrm{H}$

$\mathrm{H}$

$\mathrm{C}$

$\mathrm{H}$

$\mathrm{H}$

$\mathrm{C}$

$\mathrm{H}$

$\mathrm{C}$

$\mathrm{O}$

$\mathrm{N}$

$\mathrm{H}$

$\mathrm{H}$

$\mathrm{C}$

$\mathrm{H}$

C

$\mathrm{H}$

$\mathrm{H}$

C

$\mathrm{H}$

$\mathrm{H}$

C

$\mathrm{H}$

$\mathrm{H}$

C

$\mathrm{H}$

C

$\mathrm{O}$

$\mathrm{N}$

$\mathrm{H}$

C

$\mathrm{H}$

$\mathrm{C}$

$\mathrm{H}$

$\mathrm{H}$

C

$\mathrm{H}$

$\mathrm{H}$

$\mathrm{C}$

$\mathrm{H}$

$$
-4.70459
$$

$-5.64402$

$-3.72526$

$-2.8823$

$-4.35218$

$-4.97315$

$-3.54682$

$-4.30354$

$-3.8131$

$-4.93847$

$-5.12687$

$-5.2588$

$-6.12946$

$-3.24162$

$-2.28531$

$-3.02796$

$-3.9721$

$-6.6683$

$-7.52427$

$-5.89498$

$-6.46711$

$-7.45251$

$-5.84048$

$-6.5062$

$-4.92537$

$-5.13621$

$-4.06018$

$-5.61481$

$-5.53176$

$-6.4267$

$-4.7462$

$-5.5695$

$-6.17699$

$-4.36752$

$-3.20517$

$-0.27571$

$-1.27946$

0.643226

1.546818

0.004422

$-0.59621$

0.809512

$-0.16223$

0.189331

$-0.86833$

$-0.81502$

$-0.81215$
0.650438

$\begin{array}{ll}0.881977 & -0.83631\end{array}$

$\begin{array}{ll}1.716742 & -1.32858\end{array}$

$\begin{array}{ll}1.284384 & -1.86287\end{array}$

$2.907571 \quad-2.07134$

$2.585968-2.91022$

$3.531474-2.47111$

$\begin{array}{ll}3.473671 & 0.31956\end{array}$

$\begin{array}{ll}4.398021 & 0.632548\end{array}$

$3.147907 \quad 1.143763$

$\begin{array}{ll}3.674396 & -0.98232\end{array}$

$4.728053-1.23654$

$\begin{array}{ll}3.252271 & -0.86352\end{array}$

$\begin{array}{ll}2.385882 & 0.004331\end{array}$

$\begin{array}{ll}2.860375 & -0.23083\end{array}$

$1.390928 \quad 1.138359$

$0.95438 \quad 1.802373$

$0.114525 \quad 0.727211$

$0.238831 \quad 1.255289$

$\begin{array}{ll}0.470254 & 1.291616\end{array}$

$\begin{array}{ll}-1.30368 & 0.419231\end{array}$

$\begin{array}{ll}-1.71694 & 0.174695\end{array}$

$\begin{array}{ll}-2.18557 & 1.51307\end{array}$

$\begin{array}{ll}-2.31411 & 2.371191\end{array}$

$-1.703391 .872857$

$-3.07479 \quad-0.66211$

$-3.14714 \quad-0.8181$

$-3.71446-1.40659$

$\begin{array}{ll}-3.49825 & 0.781649\end{array}$

$-4.128420 .761086$

$\begin{array}{ll}-4.07983 & 1.269029\end{array}$

$\begin{array}{ll}-1.59952 & -0.83541\end{array}$

$-1.46413-1.73483$

$-0.65162-1.01795$

$-1.06051-1.10121$

$-1.10051 \quad-0.62252$

$-1.06405-0.79316$

$-1.02816-1.75587$

$\begin{array}{ll}-1.55927 & -1.46267\end{array}$

$-1.61002-3.02822$

$-2.49638-2.81436$

$-1.91126-3.70446$

$0.855938-3.1216$

$1.499338-3.93118$

$\begin{array}{ll}1.440227 & -2.53095\end{array}$

$-0.45398 \quad-3.64699$

$-0.5047 \quad-4.73781$
$\mathrm{H}$

C

$\mathrm{O}$

$\mathrm{N}$

$\mathrm{H}$

C

$\mathrm{H}$

C

$\mathrm{H}$

$\mathrm{H}$

C

H

$\mathrm{H}$

C

$\mathrm{H}$

$\mathrm{H}$

$\mathrm{C}$

$\mathrm{H}$

$\mathrm{C}$

$\mathrm{O}$

$\mathrm{N}$

$\mathrm{H}$

C

$\mathrm{H}$

C

H

$\mathrm{H}$

C

$\mathrm{H}$

$\mathrm{H}$

C

$\mathrm{H}$

$\mathrm{H}$

C

$\mathrm{H}$

C

N

H

$\mathrm{H}$

$\mathrm{O}$

$\mathrm{N}$

$\mathrm{H}$

C

$\mathrm{H}$

C

$\mathrm{H}$

$\mathrm{H}$

C

$\mathrm{H}$

$\mathrm{H}$

$\begin{array}{rrr}-1.85545 & -0.5189 & -3.3238 \\ 1.012396 & 0.418387 & -2.21978 \\ 1.916278 & 0.30156 & -2.82226 \\ 1.309475 & 1.371499 & -1.07274 \\ 0.414935 & 1.916513 & -0.41874 \\ -1.74449 & 1.014462 & 1.344035 \\ -1.01938 & 1.382347 & 0.729305 \\ -1.38645 & -0.00667 & 2.306797 \\ -2.29536 & -0.20527 & 2.877217 \\ -0.25341 & 0.374543 & 3.272715 \\ -0.58657 & 1.106764 & 4.011299 \\ 0.566723 & 0.823808 & 2.707363 \\ -0.38237 & -2.08813 & 2.971424 \\ 0.373711 & -2.83064 & 2.718058 \\ -1.20716 & -2.60891 & 3.464984 \\ 0.184979 & -0.97016 & 3.898013 \\ 1.271498 & -1.03276 & 3.966074 \\ -0.20874 & -1.07773 & 4.911806 \\ -0.92464 & -1.37747 & 1.715149 \\ -1.7803 & -1.89195 & 1.273368 \\ 0.15978 & -1.22618 & 0.648256 \\ 1.362666 & -1.23297 & 0.946674 \\ 4.273006 & -0.67794 & 0.858237 \\ 3.270993 & -0.86405 & 0.877928 \\ 5.20444 & -1.76309 & 0.558134 \\ 6.144967 & -1.48663 & 1.033898 \\ 4.679083 & -3.12138 & 1.049674 \\ 4.185544 & -3.03906 & 2.020172 \\ 5.530925 & -3.80005 & 1.164924 \\ 4.431512 & -3.1334 & -1.38285 \\ 4.973618 & -3.9485 & -1.86813 \\ 3.70735 & -2.75294 & -2.10317 \\ 3.754769 & -3.61401 & -0.0756 \\ 3.614379 & -4.69701 & -0.05345 \\ 2.769159 & -3.15703 & 0.037419 \\ 5.423737 & -2.01216 & -0.97973 \\ 6.451951 & -2.36634 & -1.09777 \\ 5.288531 & -0.73042 & -1.79854 \\ 6.356532 & 0.114351 & -1.72962 \\ 6.215443 & 1.023818 & -2.14708 \\ 6.906114 & 0.116628 & -0.87976 \\ 4.301576 & -0.4692 & -2.47995 \\ 2.625582 & 1.567741 & -0.81512 \\ 3.289783 & 1.052719 & -1.38391 \\ 3.094841 & 2.364343 & 0.302314 \\ 2.216929 & 2.907802 & 0.655645 \\ 4.209488 & 3.367161 & -0.03703 \\ 3.827474 & 4.207112 & -0.62107 \\ 4.979158 & 2.871651 & -0.63586 \\ 4.252705 & 2.743717 & 2.36721 \\ 5.03851 & 2.39456 & 3.036185 \\ 3.461633 & 3.184404 & 2.98002 \\ & & \end{array}$




\begin{tabular}{|c|c|c|c|c|c|c|c|}
\hline $\mathrm{C}$ & 4.785591 & 3.784601 & 1.337896 & $\mathrm{H}$ & -4.94771 & -0.74655 & 0.110295 \\
\hline $\mathrm{H}$ & 5.875138 & 3.779962 & 1.315715 & $\mathrm{H}$ & -2.41142 & -0.58787 & -1.18442 \\
\hline $\mathrm{H}$ & 4.467286 & 4.79404 & 1.609302 & $\mathrm{H}$ & -2.5363 & -2.95685 & -1.29942 \\
\hline $\mathrm{C}$ & 3.654366 & 1.590148 & 1.539434 & $\mathrm{H}$ & -4.24848 & -2.59532 & -1.06536 \\
\hline $\mathrm{H}$ & 2.83823 & 1.078026 & 2.05292 & $\mathrm{H}$ & -3.12029 & -4.31872 & 0.667828 \\
\hline $\mathrm{C}$ & 4.726353 & 0.56528 & 1.166577 & $\mathrm{H}$ & -4.0203 & -2.98076 & 1.377812 \\
\hline \multirow[t]{4}{*}{$\mathrm{O}$} & 5.923428 & 0.859157 & 1.137402 & $\mathrm{H}$ & -0.98714 & -3.17375 & 0.968538 \\
\hline & & $\mathrm{H}$ & -1.82802 & -2.70732 & 2.441207 \\
\hline & & 4: H8 & & $\mathrm{H}$ & -2.11239 & -0.49074 & 1.644059 \\
\hline & \multicolumn{3}{|c|}{ B3LYP/6-311G**: -2241.54851631 a. u. } & $\mathrm{N}$ & -0.29126 & 0.56944 & 0.122075 \\
\hline $\mathrm{N}$ & -10.5589 & 1.137296 & -0.45315 & $\mathrm{C}$ & 0.925542 & 1.155997 & -0.44238 \\
\hline $\mathrm{H}$ & -11.0596 & 1.295279 & 0.418353 & $\mathrm{C}$ & 0.660273 & 2.615029 & -0.85554 \\
\hline $\mathrm{H}$ & -11.156 & 1.474597 & -1.20197 & $\mathrm{C}$ & 0.982118 & 3.433113 & 0.403129 \\
\hline $\mathrm{C}$ & -10.2696 & -0.29265 & -0.61948 & $\mathrm{C}$ & 2.251434 & 2.754569 & 0.94302 \\
\hline $\mathrm{C}$ & -11.4159 & -1.29958 & -0.43162 & $\mathrm{C}$ & 2.08918 & 1.252065 & 0.617714 \\
\hline $\mathrm{C}$ & -10.6831 & -2.64225 & -0.29234 & $\mathrm{C}$ & 3.387383 & 0.594344 & 0.128903 \\
\hline $\mathrm{C}$ & -9.3907 & -2.31347 & 0.503615 & $\mathrm{O}$ & 4.42029 & 1.239822 & -0.07566 \\
\hline $\mathrm{C}$ & -9.19968 & -0.78109 & 0.395077 & $\mathrm{H}$ & -1.14771 & 1.125119 & 0.145987 \\
\hline $\mathrm{C}$ & -7.77436 & -0.33952 & 0.052141 & $\mathrm{H}$ & 1.233052 & 0.541283 & -1.29179 \\
\hline $\mathrm{O}$ & -6.81947 & -1.11995 & 0.080245 & $\mathrm{H}$ & -0.35443 & 2.758677 & -1.2343 \\
\hline $\mathrm{H}$ & -9.86614 & -0.4136 & -1.63225 & $\mathrm{H}$ & 1.355484 & 2.883956 & -1.65665 \\
\hline $\mathrm{H}$ & -12.1374 & -1.27724 & -1.25304 & $\mathrm{H}$ & 0.159179 & 3.355273 & 1.121421 \\
\hline $\mathrm{H}$ & -11.9576 & -1.05829 & 0.492151 & $\mathrm{H}$ & 1.129367 & 4.494704 & 0.191312 \\
\hline $\mathrm{H}$ & -10.426 & -3.02347 & -1.28552 & $\mathrm{H}$ & 2.405785 & 2.926394 & 2.010331 \\
\hline $\mathrm{H}$ & -11.2909 & -3.40761 & 0.193532 & $\mathrm{H}$ & 3.137296 & 3.127793 & 0.424814 \\
\hline $\mathrm{H}$ & -8.51679 & -2.8335 & 0.112272 & $\mathrm{H}$ & 1.770781 & 0.696378 & 1.501983 \\
\hline $\mathrm{H}$ & -9.48855 & -2.60827 & 1.550289 & $\mathrm{~N}$ & 3.303008 & -0.73812 & -0.05908 \\
\hline $\mathrm{H}$ & -9.43755 & -0.312 & 1.358907 & $\mathrm{H}$ & 2.393916 & -1.1763 & 0.09713 \\
\hline $\mathrm{N}$ & -7.64666 & 0.969221 & -0.25872 & $\mathrm{C}$ & 4.398886 & -1.53893 & -0.59579 \\
\hline $\mathrm{C}$ & -6.37194 & 1.564034 & -0.66568 & $\mathrm{C}$ & 3.882418 & -2.91128 & -1.09112 \\
\hline $\mathrm{C}$ & -6.5908 & 3.015214 & -1.12732 & $\mathrm{C}$ & 4.994083 & -3.90806 & -0.73829 \\
\hline $\mathrm{C}$ & -6.46222 & 3.847836 & 0.155756 & $\mathrm{C}$ & 5.499423 & -3.39889 & 0.619134 \\
\hline $\mathrm{C}$ & -5.27116 & 3.191906 & 0.873826 & $\mathrm{C}$ & 5.531359 & -1.86872 & 0.45297 \\
\hline $\mathrm{C}$ & -5.36411 & 1.685093 & 0.541222 & $\mathrm{C}$ & 6.92003 & -1.37232 & 0.00625 \\
\hline $\mathrm{C}$ & -4.0027 & 1.046833 & 0.229871 & $\mathrm{O}$ & 7.882519 & -2.13813 & -0.10234 \\
\hline $\mathrm{O}$ & -2.96832 & 1.713684 & 0.125063 & $\mathrm{H}$ & 4.837049 & -0.97862 & -1.42511 \\
\hline $\mathrm{H}$ & -8.51145 & 1.503721 & -0.34164 & $\mathrm{H}$ & 3.630095 & -2.89045 & -2.15378 \\
\hline $\mathrm{H}$ & -5.94966 & 0.943487 & -1.45925 & $\mathrm{H}$ & 2.969136 & -3.17345 & -0.54655 \\
\hline $\mathrm{H}$ & -7.53871 & 3.144249 & -1.65657 & $\mathrm{H}$ & 5.805766 & -3.85793 & -1.47105 \\
\hline $\mathrm{H}$ & -5.78974 & 3.285519 & -1.82156 & $\mathrm{H}$ & 4.639056 & -4.94089 & -0.708 \\
\hline $\mathrm{H}$ & -7.3749 & 3.761129 & 0.755471 & $\mathrm{H}$ & 6.480044 & -3.79038 & 0.88614 \\
\hline $\mathrm{H}$ & -6.30312 & 4.910079 & -0.04229 & $\mathrm{H}$ & 4.787566 & -3.66957 & 1.405848 \\
\hline $\mathrm{H}$ & -5.27094 & 3.372944 & 1.950551 & $\mathrm{H}$ & 5.301808 & -1.35534 & 1.390135 \\
\hline $\mathrm{H}$ & -4.32729 & 3.575638 & 0.480987 & $\mathrm{~N}$ & 7.00522 & -0.04949 & -0.2361 \\
\hline $\mathrm{H}$ & -5.78797 & 1.128127 & 1.378944 & $\mathrm{C}$ & 8.241923 & 0.581168 & -0.70095 \\
\hline $\mathrm{N}$ & -4.03376 & -0.29214 & 0.079313 & $\mathrm{C}$ & 7.971934 & 2.040343 & -1.1066 \\
\hline $\mathrm{C}$ & -2.85722 & -1.07015 & -0.31121 & $\mathrm{C}$ & 8.151344 & 2.835569 & 0.194027 \\
\hline $\mathrm{C}$ & -3.26125 & -2.52565 & -0.60249 & $\mathrm{C}$ & 9.383307 & 2.177496 & 0.840543 \\
\hline $\mathrm{C}$ & -3.15366 & -3.23052 & 0.756805 & $\mathrm{C}$ & 9.323014 & 0.68625 & 0.440274 \\
\hline $\mathrm{C}$ & -1.86181 & -2.6439 & 1.351554 & $\mathrm{C}$ & 10.6813 & 0.128736 & -0.0189 \\
\hline $\mathrm{C}$ & -1.80286 & -1.18059 & 0.856765 & $\mathrm{~N}$ & 10.68284 & -1.22052 & -0.1834 \\
\hline $\mathrm{C}$ & -0.402 & -0.74429 & 0.406637 & $\mathrm{H}$ & 11.50871 & -1.64012 & -0.58001 \\
\hline $\mathrm{O}$ & 0.539334 & -1.53727 & 0.306201 & $\mathrm{H}$ & 9.808582 & -1.73976 & -0.15599 \\
\hline
\end{tabular}




$\begin{array}{lrrr}\mathrm{O} & 11.66197 & 0.829167 & -0.21803 \\ \mathrm{H} & 6.154422 & 0.511813 & -0.16349 \\ \mathrm{H} & 8.62265 & -0.01208 & -1.53517 \\ \mathrm{H} & 6.991926 & 2.165261 & -1.57337 \\ \mathrm{H} & 8.731224 & 2.345533 & -1.83312 \\ \mathrm{H} & 7.264883 & 2.71953 & 0.826503 \\ \mathrm{H} & 8.287675 & 3.905729 & 0.019432 \\ \mathrm{H} & 9.412882 & 2.308334 & 1.924572 \\ \mathrm{H} & 10.30596 & 2.599891 & 0.43569 \\ \mathrm{H} & 8.987468 & 0.065568 & 1.272929\end{array}$


Ref. 16: Gaussian 03, Revision A.1, Frisch, M. J.; Trucks, G. W.; Schlegel, H. B.; Scuseria, G. E.; Robb, M. A.; Cheeseman, J. R.; Montgomery, Jr., J. A.; Vreven, T.; Kudin, K. N.; Burant, J. C.; Millam, J. M.; Iyengar, S. S.; Tomasi, J.; Barone, V.; Mennucci, B.; Cossi, M.; Scalmani, G.; Rega, N.; Petersson, G. A.; Nakatsuji, H.; Hada, M.; Ehara, M.; Toyota, K.; Fukuda, R.; Hasegawa, J.; Ishida, M.; Nakajima, T.; Honda, Y.; Kitao, O.; Nakai, H.; Klene, M.; Li, X.; Knox, J. E.; Hratchian, H. P.; Cross, J. B.; Adamo, C.; Jaramillo, J.; Gomperts, R.; Stratmann, R. E.; Yazyev, O.; Austin, A. J.; Cammi, R.; Pomelli, C.; Ochterski, J. W.; Ayala, P. Y.; Morokuma, K.; Voth, G. A.; Salvador, P.; Dannenberg, J. J.; Zakrzewski, V. G.; Dapprich, S.; Daniels, A. D.; Strain, M. C.; Farkas, O.; Malick, D. K.; Rabuck, A. D.; Raghavachari, K.; Foresman, J. B.; Ortiz, J. V.; Cui, Q.; Baboul, A. G.; Clifford, S.; Cioslowski, J.; Stefanov, B. B.; Liu, G.; Liashenko, A.; Piskorz, P.; Komaromi, I.; Martin, R. L.; Fox, D. J.; Keith, T.; Al-Laham, M. A.; Peng, C. Y.; Nanayakkara, A.; Challacombe, M.; Gill, P. M. W.; Johnson, B.; Chen, W.; Wong, M. W.; Gonzalez, C.; and Pople, J. A.; Gaussian, Inc., Pittsburgh PA, 2003. http://www.gaussian.com 